PROCEEDINGS OF THE

AMERICAN MATHEMATICAL SOCIETY

Volume 128, Number 12, Pages 3519-3522

S 0002-9939(00)05907-4

Article electronically published on July 6, 2000

\title{
TRACE-CLASS PERTURBATION AND STRONG CONVERGENCE: WAVE OPERATORS REVISITED
}

\author{
JINGBO XIA
}

(Communicated by David R. Larson)

\begin{abstract}
We give a new construction of wave operators for a self-adjoint operator under trace-class perturbation. This construction requires no quantitative estimates.
\end{abstract}

The well-known theorem of Kato and Rosenblum [2, 5] asserts that if $A$ and $A^{\prime}$ are self-adjoint operators and if $A-A^{\prime}$ belongs to $\mathcal{C}_{1}$, the trace class, then the absolutely continuous parts of $A$ and $A^{\prime}$ are unitarily equivalent. Because of the importance of the problem of trace-class perturbation, many improvements and generalizations of this theorem have appeared in the literature. 3] and 4] contain a reasonably complete account of the development between [2], 5] and the late 1970 's. In these works the unitary equivalence between $A_{\mathrm{ac}}$ and $A_{\mathrm{ac}}^{\prime}$ was established through the existence of the wave operators

$$
W_{ \pm}\left(A^{\prime}, A\right)=\mathrm{s}^{-} \lim _{\lambda \rightarrow \pm \infty} e^{-i \lambda A^{\prime}} e^{i \lambda A} P_{\mathrm{ac}}(A) .
$$

In fact, as mentioned in [5], the original idea of using the operator $e^{-i \lambda A^{\prime}} e^{i \lambda A}$ dates back to Friedrichs 1. The first generalization of the Kato-Rosenblum theorem to the setting of operator tuples was made by Voigt 8 . The use of the exponential function $e_{\lambda}(x)=\exp (i \lambda x)$ is fundamental to these "time-dependent" constructions of wave operators.

In [6], Voiculescu generalized wave operators to the setting of commuting tuples under perturbation by norm ideals of compact operators. He showed that, if $T=$ $\left(T_{1}, \ldots, T_{N}\right)$ and $T^{\prime}=\left(T_{1}^{\prime}, \ldots, T_{N}^{\prime}\right)$ are commuting tuples of self-adjoint operators such that $T_{j}-T_{j}^{\prime} \in \mathcal{C}^{(0)}, j=1, \ldots, N$, and if the norm ideal $\mathcal{C}$ has the property

$$
\lim _{n \rightarrow \infty} n^{-1 / 2}\left\|\omega_{1} \otimes \omega_{1}+\cdots+\omega_{n} \otimes \omega_{n}\right\|_{\mathcal{C}}=0,
$$

where $\left\{\omega_{n}\right\}_{n=1}^{\infty}$ is any orthonormal set, then the wave operator for the perturbation problem $T \rightarrow T^{\prime}$ exists in the strong operator topology and is unique [6, Theorem 1.5]. This uniqueness is in sharp contrast with the problem of trace-class perturbation for single operator; in general, the two wave operators $W_{+}$and $W_{-}$do not necessarily coincide. While essentially a time-dependent approach, Voiculescu's

Received by the editors January 26, 1999.

2000 Mathematics Subject Classification. Primary 47A40, 47B10, 47L20.

This research was supported in part by National Science Foundation grant DMS-9703515.

(C)2000 American Mathematical Society 
work shows that, for perturbations other than that by the trace class, it is possible to construct wave operators without explicitly using the exponential function $e_{\lambda}(x)=\exp (i \lambda x)$.

The treatment of the original theorem of Kato-Rosenblum in the framework of [6], however, proved to be elusive. In fact this was one of the problems Voiculescu raised during the 1983 ICM [7, page 1043]. Also, the reason for the fact that $W_{+}$ and $W_{-}$may differ or, equivalently, that the scattering operator $S=W_{-}^{*} W_{+}$is not necessarily $P_{\mathrm{ac}}(A)$, has never been made clear in the previous constructions of wave operators; one usually gets $S \neq P_{\mathrm{ac}}(A)$ from explicit computations.

The purpose of this note is to give a proof of the Kato-Rosenblum theorem within the context suggested by Voiculescu [6], 7]. Indeed, what we will prove is slightly stronger than the original version. Moreover, this new proof has the following three distinct features: (a) It identifies the cause for $W_{+} \neq W_{-}$. (b) It only uses certain limited properties satisfied by the exponential functions $e_{\lambda}(x)=\exp (i \lambda x)$, but not the exponential functions themselves. (c) Unlike previous ones, our proof involves no quantitative estimates. Indeed, our proof is surprisingly soft.

We start by recalling a few well-known facts. For the rest of the paper, $H$ and $M$ will denote the Hilbert transform and the multiplication by the coordinate function on $\mathbf{R}$. That is, for $f \in L^{2}(\mathbf{R})$ or for $f \in L^{2}(\mathbf{R}, \mathcal{M})=L^{2}(\mathbf{R}) \otimes \mathcal{M}$, where $\mathcal{M}$ is a Hilbert space, we write

$$
(H f)(x)=\frac{1}{\pi i} \text { p.v. } \int \frac{f(y)}{y-x} d y \quad \text { and } \quad(M f)(x)=x f(x) .
$$

Recall that $e^{i \lambda M} H e^{-i \lambda M}=\chi_{(0, \infty)}(D-\lambda)-\chi_{(-\infty, 0)}(D-\lambda)$, where $D$ is the differential operator $(1 / i) d / d x$. Hence

$$
\text { s- } \lim _{\lambda \rightarrow \infty} e^{i \lambda M} H e^{-i \lambda M}=-1 \quad \text { and } \quad \mathrm{s}-\lim _{\lambda \rightarrow-\infty} e^{i \lambda M} H e^{-i \lambda M}=1 .
$$

Also recall that if $A$ and $A^{\prime}$ are self-adjoint operators, $z \in \mathbf{C} \backslash \mathbf{R}$ and $\lambda \in \mathbf{R}$, then

$$
\left(A^{\prime}-z\right)^{-1}\left(e^{i \lambda A^{\prime}}-e^{i \lambda A}\right)(A-z)^{-1}=i \int_{0}^{\lambda} e^{i s A^{\prime}}\left\{(A-z)^{-1}-\left(A^{\prime}-z\right)^{-1}\right\} e^{i(\lambda-s) A} d s .
$$

Therefore if $(A-z)^{-1}-\left(A^{\prime}-z\right)^{-1}$ belongs to the trace class, then so does $\left(A^{\prime}-z\right)^{-1}\left(e^{i \lambda A^{\prime}}-e^{i \lambda A}\right)(A-z)^{-1}$. As it turns out, these are the only properties of the exponential function which are relevant to the construction of wave operators.

A sequence of Borel functions $\left\{\varphi_{n}\right\}$ on $\mathbf{R}$ is said to be of class $\Omega_{+}$if

(i) $\left|\varphi_{n}(t)\right|=1$ for all $t \in \mathbf{R}$ and $n \in \mathbf{N}$.

(ii) If $A$ and $A^{\prime}$ are self-adjoint operators such that $(A-z)^{-1}-\left(A^{\prime}-z\right)^{-1} \in \mathcal{C}_{1}$ for some $z \in \mathbf{C} \backslash \mathbf{R}$, then $\left(A^{\prime}-z\right)^{-1}\left(\varphi_{n}\left(A^{\prime}\right)-\varphi_{n}(A)\right)(A-z)^{-1} \in \mathcal{C}_{1}$ for every $n \in \mathbf{N}$. (iii $\left.{ }^{+}\right) \mathrm{s}^{-} \lim _{n \rightarrow \infty} \varphi_{n}(M) H \varphi_{n}^{*}(M)=-1$.

A sequence of Borel functions $\left\{\varphi_{n}\right\}$ on $\mathbf{R}$ is said to be of class $\Omega_{-}$if it satisfies (i), (ii) and

(iii $\left.{ }^{-}\right) \mathrm{s}^{-} \lim _{n \rightarrow \infty} \varphi_{n}(M) H \varphi_{n}^{*}(M)=1$.

It is elementary that (ii) and (i) imply

(II) If $A$ and $A^{\prime}$ are self-adjoint operators such that $(A-z)^{-1}-\left(A^{\prime}-z\right)^{-1} \in \mathcal{C}_{1}$ for some $z \in \mathbf{C} \backslash \mathbf{R}$, then $\left(\varphi_{n}\left(A^{\prime}\right)-\varphi_{n}(A)\right)(A-z)^{-2} \in \mathcal{C}_{1}$ for every $n \in \mathbf{N}$.

Because $\left\|(H \pm 1) \varphi_{n}^{*}(M) f\right\|=\left\|\varphi_{n}(M)(H \pm 1) \varphi_{n}^{*}(M) f\right\|,\left(\right.$ iii $\left.^{+}\right)$and (iii ${ }^{-}$) respectively imply

$\left(\mathrm{III}^{+}\right) \mathrm{s}-\lim _{n \rightarrow \infty}(H+1) \varphi_{n}^{*}(M)=0$.

$\left(\mathrm{III}^{-}\right) \mathrm{s}-\lim _{n \rightarrow \infty}(H-1) \varphi_{n}^{*}(M)=0$. 
Thus, if $\left\{\lambda_{n}\right\}$ are positive numbers such that $\lim _{n \rightarrow \infty} \lambda_{n}=\infty$, then the sequence $\left\{\exp \left(i \lambda_{n} x\right)\right\}\left(\right.$ resp. $\left.\left\{\exp \left(-i \lambda_{n} x\right)\right\}\right)$ is of class $\Omega_{+}$(resp. $\left.\Omega_{-}\right)$. As we will see, the dichotomy between ( $\mathrm{iii}^{+}$) and (iii ${ }^{-}$) is the cause for $W_{+} \neq W_{-}$.

Theorem. Let $A$ and $A^{\prime}$ be self-adjoint operators on a Hilbert space $\mathcal{H}$ such that

$$
(A-z)^{-1}-\left(A^{\prime}-z\right)^{-1} \in \mathcal{C}_{1} \quad \text { for some } z \in \mathbf{C} \backslash \mathbf{R} .
$$

Then there are partial isometries $W_{+}\left(A^{\prime}, A\right)$ and $W_{-}\left(A^{\prime}, A\right)$ such that

$$
\begin{aligned}
s-\lim _{n \rightarrow \infty} \varphi_{n}^{*}\left(A^{\prime}\right) \varphi_{n}(A) P_{a c}(A) & =W_{+}\left(A^{\prime}, A\right) \quad \text { and } \\
s-\lim _{n \rightarrow \infty} \psi_{n}^{*}\left(A^{\prime}\right) \psi_{n}(A) P_{a c}(A) & =W_{-}\left(A^{\prime}, A\right),
\end{aligned}
$$

where $\left\{\varphi_{n}\right\}$ is any sequence of class $\Omega_{+}$and $\left\{\psi_{n}\right\}$ any sequence of class $\Omega_{-}$.

Proof. We only need to establish the strong convergence; that the limits $W_{+}\left(A^{\prime}, A\right)$ and $W_{-}\left(A^{\prime}, A\right)$ are independent of the choices of $\left\{\varphi_{n}\right\}$ and $\left\{\psi_{n}\right\}$ follows from an observation borrowed from [6]: If one mixes two sequences of a given class, then one obtains a new sequence of the same class. Moreover, we will only consider the case of $\Omega_{+}$; the case of $\Omega_{-}$differs only in one detail, which will be pointed out in due course.

We may assume that $\mathcal{H}=\left(\bigoplus_{j \in J} L^{2}\left(\Delta_{j}\right)\right) \oplus \mathcal{H}_{\mathrm{s}}$, where each summand is invariant under $A, A \mid \mathcal{H}_{\mathrm{s}}$ is purely singular, and $A \mid\left(\bigoplus_{j \in J} L^{2}\left(\Delta_{j}\right)\right)=M$, the multiplication by the coordinate function. Here, each $\Delta_{j}$ is a Borel set in $\mathbf{R}$ and, as usual, $L^{2}\left(\Delta_{j}\right)=\chi_{\Delta_{j}} L^{2}(\mathbf{R})$. Define $W_{n}=\varphi_{n}^{*}\left(A^{\prime}\right) \varphi_{n}(A)$ and $T_{k, n}=W_{k}^{*} W_{n}-1$ for $n$, $k \in \mathbf{N}$.

Pick a $j_{0} \in J$ and let $\xi \in L^{2}\left(\Delta_{j_{0}}\right)$ be a bounded function whose support is contained in a finite interval $I$. Define the operator $\tilde{M}_{\xi}$ on $\mathcal{H}$ by the formula $\tilde{M}_{\xi} f=\xi f_{j_{0}}$ for $f=\left(\bigoplus_{j \in J} f_{j}\right) \oplus h$, where $h \in \mathcal{H}_{\mathrm{s}}$ and $f_{j} \in L^{2}\left(\Delta_{j}\right), j \in J$. Define

$$
Y_{\xi}=\pi i(A-z) \tilde{M}_{\xi}(H+1) \tilde{M}_{\xi}^{*}(A-z) .
$$

(For the case of $\Omega_{-}$, replace the operator $H+1$ above by $H-1$.) Because $\operatorname{supp} \xi \subset$ $I, Y_{\xi}$ is a bounded operator. Denote $K=(A-z)^{-1}-\left(A^{\prime}-z\right)^{-1}$. We have $\left[T_{k, n},(A-z)^{-1}\right]=W_{k}^{*} \varphi_{n}^{*}\left(A^{\prime}\right) K \varphi_{n}(A)-\varphi_{k}^{*}(A) K \varphi_{k}\left(A^{\prime}\right) W_{n} \in \mathcal{C}_{1}$ and, therefore,

$$
\left|\operatorname{tr}\left(\left[T_{k, n},(A-z)^{-1}\right] Y_{\xi}\right)\right| \leq\left\|K \varphi_{n}(A) Y_{\xi}\right\|_{1}+\left\|Y_{\xi} \varphi_{k}^{*}(A) K\right\|_{1} .
$$

It follows from $\left(\mathrm{III}^{+}\right)$, the identity $\tilde{M}_{\xi}^{*} \varphi_{n}^{*}(A)=\varphi_{n}^{*}(M) \tilde{M}_{\xi}^{*}$, and the assumptions on $\xi$ that $\mathrm{s}-\lim _{n \rightarrow \infty} Y_{\xi}^{*} \varphi_{n}^{*}(A)=0=\mathrm{s}-\lim _{k \rightarrow \infty} Y_{\xi} \varphi_{k}^{*}(A)$, which leads to $\left\|Y_{\xi} \varphi_{k}^{*}(A) K\right\|_{1} \rightarrow 0$ and $\left\|K \varphi_{n}(A) Y_{\xi}\right\|_{1}=\left\|Y_{\xi}^{*} \varphi_{n}^{*}(A) K^{*}\right\|_{1} \rightarrow 0$ as $\min \{k, n\} \rightarrow \infty$. That is,

$$
\lim _{\min \{k, n\} \rightarrow \infty} \operatorname{tr}\left(\left[T_{k, n},(A-z)^{-1}\right] Y_{\xi}\right)=0 .
$$

By (II), $\left(W_{n}-1\right)(A-z)^{-2} \in \mathcal{C}_{1}$ and $\left(W_{k}^{*}-1\right)(A-z)^{-2} \in \mathcal{C}_{1}$. Since $T_{k, n}=$ $\left(W_{k}^{*}-1\right)\left(W_{n}-1\right)+\left(W_{k}^{*}-1\right)+\left(W_{n}-1\right)$, we have $T_{k, n}(A-z)^{-2} \in \mathcal{C}_{1}$. Since $(A-z)^{2} Y_{\xi}$ is bounded, $T_{k, n} Y_{\xi}=\left\{T_{k, n}(A-z)^{-2}\right\}\left\{(A-z)^{2} Y_{\xi}\right\} \in \mathcal{C}_{1}$. Thus, $\operatorname{tr}\left((A-z)^{-1} T_{k, n} Y_{\xi}\right)$ $=\operatorname{tr}\left(T_{k, n} Y_{\xi}(A-z)^{-1}\right)$. Now $Y_{\xi}$ was designed so that $\left[(A-z)^{-1}, Y_{\xi}\right]=\xi \otimes \xi$. Hence

$$
\operatorname{tr}\left(\left[T_{k, n},(A-z)^{-1}\right] Y_{\xi}\right)=\operatorname{tr}\left(T_{k, n}\left[(A-z)^{-1}, Y_{\xi}\right]\right)=\operatorname{tr}\left(T_{k, n} \xi \otimes \xi\right)=\left\langle T_{k, n} \xi, \xi\right\rangle
$$


Because $\left(W_{k}-W_{n}\right)^{*}\left(W_{k}-W_{n}\right)=-T_{k, n}-T_{n, k}$, it follows from (1) and (2) that

$$
\lim _{\min \{k, n\} \rightarrow \infty}\left\|\left(W_{k}-W_{n}\right) \xi\right\|^{2}=-\lim _{\min \{k, n\} \rightarrow \infty}\left\langle\left(T_{k, n}+T_{n, k}\right) \xi, \xi\right\rangle=0 .
$$

Since the linear span of such $\xi$ 's is dense in $\bigoplus_{j \in J} L^{2}\left(\Delta_{j}\right)$, this completes the proof.

Remark. To deduce that $\left(A^{\prime}-w\right)^{-1} W_{+}\left(A^{\prime}, A\right)=W_{+}\left(A^{\prime}, A\right)(A-w)^{-1}$ for $w \in \mathbf{C} \backslash \mathbf{R}$, which is necessary for establishing the unitary equivalence of $A_{\mathrm{ac}}$ and $A_{\mathrm{ac}}^{\prime}$, one needs one sequence of class $\Omega_{+}$which has the additional property

$$
\text { w- } \lim _{n \rightarrow \infty} \varphi_{n}(A) P_{\mathrm{ac}}(A)=0 .
$$

For example, use the sequence $\{\exp (i n x)\}$. But, nowhere in our proof did we need anything like (3) for the strong convergence of $\left\{\varphi_{n}^{*}\left(A^{\prime}\right) \varphi_{n}(A) P_{\mathrm{ac}}(A)\right\}$. In fact it is not even clear that $\Omega_{+}$would imply (3), although it is difficult to imagine that $\left(\mathrm{iii}^{+}\right)$and $(3)$ are completely unrelated.

\section{REFERENCES}

1. K. Friedrichs, On the perturbation of continuous spectra, Commun. on Appl. Math. 1 (1948), 361-401. MR 10:547e

2. T. Kato, Perturbation of continuous spectra by trace class operators, Proc. Japan Acad. 33 (1957), 260-264. MR 19:1068d

3. T. Kato, Perturbation theory for linear operators, Springer-Verlag, New York, 1976. MR 53:11389

4. M. Reed and B. Simon, Methods of modern mathematical physics, III Scattering theory, Academic Press, New York, 1979. MR 80m:81085

5. M. Rosenblum, Perturbations of continuous spectrum and unitary equivalence, Pacific J. Math. 7 (1957), 997-1010. MR 19:756i

6. D. Voiculescu, Some results on norm-ideal perturbations of Hilbert space operators. II, J. Operator Theory 5 (1981), 77-100. |MR 83f:47014

7. D. Voiculescu, Hilbert space operators modulo normed ideals, Proc. International Congress of Mathematicians (Warsaw 1983) Vol. 2, 1041-1047, PWN, Warsaw, 1984. MR 87b:47049

8. J. Voigt, Perturbation theory for commutative $m$-tuples of self-adjoint operators, J. Funct. Anal. 25 (1977), 317-334. MR 56:9301

Department of Mathematics, State University of New York at Buffalo, Buffalo, NeW YORK 14260-2900

E-mail address: jxia@acsu.buffalo.edu 